\title{
Selective reflection by helicoidal liquid crystals. Results of an exact calculation using the $4 \times 4$ characteristic matrix method
}

\author{
I. Abdulhalim, L. Benguigui and R. Weil \\ Department of Physics and Solid State Institute, Technion, Israel Institute of Technology, Haifa 32000, Israel
}

(Reçu le 17 août 1984, révisé le 21 décembre 1984, accepté le 17 janvier 1985)

\begin{abstract}
Résumé. - On présente un calcul exact de la réflexion de la lumière par un échantillon orienté de cristal liquide hélicoïdal (SmC* ou cholestérique) en utilisant la formulation matricielle $4 \times 4$ des équations de Maxwell pour les milieux anisotropes. On montre que la méthode approximative de Berreman et Scheffer est inadéquate, spécialement en ce qui concerne la région des faibles longueurs d'onde (nombre de pics, nombre de branches dans la bande de réflexion ainsi que leur dépendance avec les différents paramètres).

Abstract. - The exact calculation of the reflection from single domain aligned samples of helicoidal liquid crystals ( $\mathrm{SmC}^{*}$ or cholesterics) is performed using the $4 \times 4$ matrix formulation of Maxwell equations in anisotropic media. We show that the expansion, approximation up to the 1st order of Berreman and Scheffer, is not adequate to describe the real situation, especially concerning the short wavelength region (heights of peaks, number of branches in the reflection band and their dependence on the different parameters).
\end{abstract}

\section{Introduction.}

The problem of selective reflection by helicoidal liquid crystals has been extensively studied $[1-14,17-$ 20], but until now there existed numerous discrepancies between the different theoretical approaches, especially in the oblique incidence case. The spiralling dielectric ellipsoid model for cholesterics is one of the basic ideas common in most of these approaches. Analytic solutions for the normal incidence case were obtained by several authors [1-4], the most illuminating one being that of De Vries [1], who used the two wave approximation for cholesterics. This approximation appears in the expression derived for the rotatory power, which is infinite at the centre of the reflection band. The most accurate formulation of the theory was given by Nityananda [4]. Then Parodi [5] showed that this theory could be applied also to SmC* if one uses some effective refractive index expressions. The basic result is that circularly polarized light, having the same helicity as the media, is totally reflected without change of the polarization state if the wavelength coincides with the pitch of the helicoidal structure, while the wave with opposite helicity is transmitted without any attenuation.

The oblique incidence case, on the other hand, is more complicated and there are only numerical calculations and approximate analytic solutions.
Belyakov and Dimitrienko $[6,7]$ have applied the many wave dynamic theory of diffraction to cholesterics and obtained approximate analytical expressions for the first and second order reflections. In the first order, one sees the existence of total reflection in a small range of incident angles where light of any polarization is totally reflected at oblique incidence, but not at normal incidence. This range is of the order of $(\delta)$ - the local dielectric anisotropy - so it is difficult to observe it experimentally. The second order peak shows a splitting into three branches, while the Toupin [8] numerical calculation shows the existence of the 1st, 2nd and 3rd order lines in which each order consists of 3 distinct lines.

Dreher and Meier $[9,10]$ investigated the problem by drawing the charts of stability of a fourth order differential equation and obtained that for oblique incidence each reflection band is split into two branches.

More recently Saupe and Meier [11] extended this investigation and found triplet of bands for large incidence angles. The outer two bands are selective reflection bands while the central one is unpolarized. The characteristic exponents were classified into four types which represent the four possible eigenmodes. The discrepancy with the previous stability charts was attributed to the omission of one kind of these eigenmodes. 
Nearly at the same time and independently, Oldano et al. $[12,13]$ solved the infinite determinant similar to that of Dreher and Meier $[9,10]$ and obtained a simple form of the dispersion relation. Their stability charts and dispersion curves show the existence of triplet of bands where the central one is common to all the eigenmodes.

Patterson [14] has developed an algorithm for solving Maxwell's equations in a cholesteric planar texture. His treatment is mainly with the scattering problem in the Born approximation. However, it is difficult, as mentioned by the author, to generalize the method to the case of chiral smectic $\mathrm{C}$ phase.

The above features are not clear in the reflectance spectra calculated by Berreman and Scheffer [17-20], however, they observed the third order reflection and the existence of an additional sequence of reflection bands in $\mathrm{SmC}^{*}$ spectra at wavelengths intermediate between the bands that appear in both cholesteric and $\mathrm{SmC}^{*}$ samples with obliquely incident light.

We believe that those discrepancies between the different approaches are the result of the approximations involved. In order to get the most accurate results, we believe that the most powerful technique is the $4 \times 4$ characteristic matrix method which was first introduced by Abeles [15, 23] in the form of $2 \times 2$ matrix method for light propagation through isotropically stratified media. Teitler and Heneves [16] extended it for anisotropic media and it was developed, especially for liquid crystals, by Berreman and Scheffer [17-20]. They wrote the characteristic matrix for one molecular layer as a series expansion in powers of its thickness, which is assumed as a small parameter so that terms with high powers were ignored. In our present work it is easily seen that this approximation, up to the 1st order, is not sufficient to give the real situation and an exact computation shows some discrepancies and additional features of the reflection spectra not reported before.

\section{Formulation of the problem.}

The $\mathrm{SmC}^{*}$ structure is one in which the molecules in each layer are tilted with respect to the normal by an angle $\theta$, and this tilt direction slowly processes about a direction parallel to the layer normal resulting in a helical structure with a pitch $P$ [21], while in cholesterics it is $P / 2$ and $\theta=90^{\circ}$.

We define a local reference frame $\xi \eta \chi$, where the long molecular axis is along $o \chi$, and a reference frame $x y z$ for the smectic layer such that $z$ is the layer normal as shown in figure 1 .

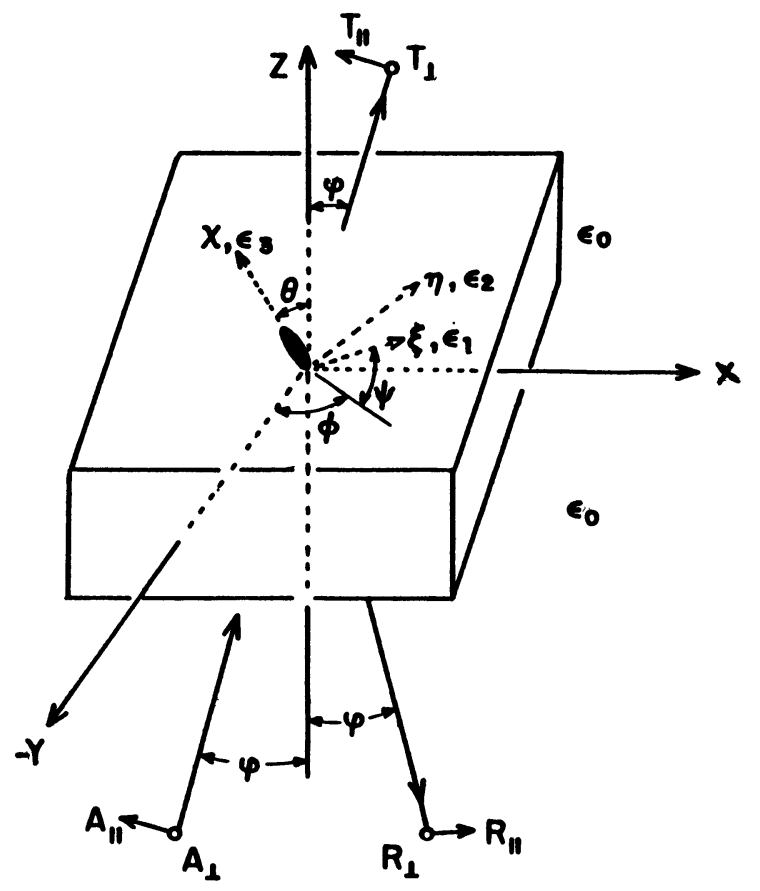

Fig. 1. - Schematic of the liquid crystal sample, sandwiched between two glass plates. The local principal dielectric tensor axes $\xi \eta \chi$ are defined by Euler angles $\theta, \phi, \psi$ where $\psi=0$. The incident plane polarized light strikes the lower plate at an angle $\varphi$. The plane of incidence is the plane of the page.

The axis $o \xi$, is a twofold symmetry axis perpendicular to $o z$ and $o \chi$. The largest principal dielectric constant $\varepsilon_{3}$ is taken along the molecular axis and in order that the principal dielectric tensor change not under symmetry operations, one of the other two principal axes has to be along $o \xi$ and the third axis $o \eta$ is chosen to be orthogonal to $o \xi$ and $o \chi$.

The angle $\phi$ is arbitrary and usually $\psi=0$. Then, one goes from the frame $(x, y, z)$ to $(\xi, \eta, \chi)$ by the rotations $\phi$ around $o z$ and $\theta$ around $o \xi$ [25], so the dielectric tensor in the $x y z$ frame is given by

$$
\hat{\varepsilon}_{x y z}=\Lambda \hat{\varepsilon}_{\xi \eta x} \tilde{\Lambda}
$$

where

$$
\hat{\varepsilon}_{\xi \eta x}=\left(\begin{array}{lll}
\varepsilon_{1} & & 0 \\
\cdot & \varepsilon_{2} & \\
0 & & \\
& & \varepsilon_{3}
\end{array}\right)
$$

and

$$
\Lambda=\left(\begin{array}{ccc}
\cos \phi-\cos \theta \sin \phi & -\cos \theta \sin \phi & \sin \theta \sin \phi \\
\sin \phi & \cos \theta \cos \phi & -\sin \theta \cos \phi \\
0 & \sin \theta & \cos \theta
\end{array}\right)
$$


The calculation shows that $\varepsilon_{x y z}$ has a periodicity equal to the full pitch while in cholesterics, which is a particular case of $\mathrm{SmC}^{*}$ when $\theta=\pi / 2$, the period equals half the full pitch.

Maxwell equations in the $4 \times 4$ matrix form [16-20] in such a medium is written as :

$$
\frac{\mathrm{d} \psi}{\mathrm{d} z}=\frac{i \omega}{c} \cdot \Delta(z) \cdot \psi
$$

where

$$
\psi=\left(\begin{array}{r}
E_{x} \\
H_{y} \\
E_{y} \\
-H_{x}
\end{array}\right)
$$

and the elements of the $\Delta$ matrix are

$$
\begin{aligned}
& \Delta_{11}=\frac{\varepsilon_{23} \cdot \sqrt{\varepsilon_{0}}}{\varepsilon_{33}} \cdot \sin \varphi \sin \phi ; \quad \Delta_{12}=1-\varepsilon_{0} \cdot \frac{\sin ^{2} \varphi}{\varepsilon_{33}} \\
& \Delta_{13}=\frac{-\varepsilon_{23} \cdot \sqrt{\varepsilon_{0}}}{\varepsilon_{33}} \cdot \sin \varphi \cos \phi ; \quad \Delta_{14}=0 \\
& \Delta_{21}=\left[\frac{\left(\varepsilon_{11}-\varepsilon_{22}\right)}{2}+\frac{\varepsilon_{23}^{2}}{2 \varepsilon_{33}}\right] \cdot \cos 2 \phi+\frac{\varepsilon_{11}+\varepsilon_{22}}{2}-\frac{\varepsilon_{23}^{2}}{2 \varepsilon_{33}} \\
& \Delta_{22}=\Delta_{11} ; \quad \Delta_{23}=\left[\frac{\left(\varepsilon_{11}-\varepsilon_{22}\right)}{2}+\frac{\varepsilon_{23}^{2}}{2 \varepsilon_{33}}\right] \cdot \sin 2 \phi ; \quad \Delta_{24}=0 \\
& \Delta_{31}=\Delta_{32}=\Delta_{33}=0 ; \quad \Delta_{34}=1.0 \\
& \Delta_{41}=\Delta_{23} ; \quad \Delta_{42}=\Delta_{13} ; \quad \Delta_{43}=\Delta_{21}-\varepsilon_{0} \cdot \sin ^{2} \varphi ; \quad \Delta_{44}=0 \\
& \varepsilon_{11}=\varepsilon_{1} ; \quad \varepsilon_{22}=\varepsilon_{2} \cdot \cos ^{2} \theta+\varepsilon_{3} \cdot \sin ^{2} \theta ; \quad \varepsilon_{23}=\left(\varepsilon_{2}-\varepsilon_{3}\right) \cdot \sin \theta \cos \theta ;
\end{aligned}
$$

$\varphi$ is the incident angle.

Equation (2) does not have an analytic solution because $\Delta$ is a function of $z$. But using the assumption that the sample may be divided in layers in which there is no change of the dielectric tensor, then $\Delta$ is also a constant of the layer. The tangential field component emerging from the $v$ th layer in terms of those entering it is given by :

The matrix

$$
\psi(h \cdot v)=\exp \left[\frac{i \cdot \omega \cdot h}{c} \cdot \Delta(h \cdot v)\right] \cdot \psi(h \cdot(v-1)) .
$$

$$
M(h \cdot v)=\exp \left[\frac{-i . \omega . h}{c} \cdot \Delta(h \cdot v)\right]
$$

is called the characteristic matrix of the layer [23], since from it one can obtain most of the optical properties of the layer.

For a pile of $m$ layers, we obtain :

$$
\psi(m h)=\left(\prod_{v=1}^{m} \exp \left[\frac{i . \omega . h}{c} \cdot \Delta(h \cdot v)\right]\right) \cdot \psi(0)
$$

where $v$ is the label number of the layer so that

$$
M(m h)=\prod_{v=1}^{m} M(h \cdot v)=\prod_{v=1}^{m} \exp \left[\frac{-i \cdot \omega \cdot h}{c} \cdot \Delta(h \cdot v)\right] .
$$

For our periodic medium with $I=P / h$ layers in one period and $N$ periods in the whole sample, the characteristic matrix is given by :

$$
M(N I h)=\left[\prod_{v=1}^{I} \exp \left(\frac{-i . \omega . h}{c} \cdot \Delta(h \cdot v)\right)\right]^{N}
$$


Berreman and Scheffer [17-18] calculated the one layer matrix $M(h \cdot v)$ by expanding the exponent in powers of the layer thickness $h$ and ignoring the terms with powers higher than the first [17] and the second [18]. The first term of the series does not converge. However, they do not show that the series converges when the second term is included. We think that the 1st order approximation is responsible for the discrepancies we. find with their work [17], while we made the exact calculation.

In order to perform the exact evaluation of $M(h \cdot v)$ one can diagonalize it numerically, then

$$
M(h \cdot v)=V \cdot\left|\begin{array}{rr}
\exp \left(\frac{-2 \pi i l_{1}}{I \lambda^{\prime}}\right) & 0 \\
\exp \left(\frac{-2 \pi i l_{2}}{I \lambda^{\prime}}\right) \\
\exp \left(\frac{-2 \pi i l_{3}}{I \lambda^{\prime}}\right) \\
0 & \exp \left(\frac{-2 \pi i l_{4}}{I \lambda^{\prime}}\right)
\end{array}\right| \cdot V^{-1}
$$

where $\lambda^{\prime}=\lambda_{0} / P$ is the reduced wavelength and $l_{j}, j=1,2,3,4$ are the eigenvalues of the $\Delta(h \cdot v)$ matrix and $V$ is the eigenvectors matrix.

Such a computation was performed recently [22] for cholesterics in order to compare it with experimental results. An additional feature was obtained which had not been reported before, that is the appearance of beats superimposed to the subsidiary oscillation if the incident light is left circularly polarized, and swells if it is right circularly polarized.

In our calculation we made use of the Lagrange-Sylvester interpolation polynomial which states that, if $l_{1}, l_{2}, l_{3}, l_{4}$ are distinct eigenvalues of any matrix $\Delta$, then any defined function of $\Delta$ is given by [24] :

$$
f(\Delta)=\sum_{i=1}^{4} f\left(l_{i}\right) \cdot \prod_{j \neq i} \frac{\left(\Delta-l_{j} \cdot I_{4}\right)}{\left(l_{i}-l_{j}\right)}
$$

where $I_{4}$ is a $4 \times 4$ unit matrix.

The evaluation of eigenvalues is given by the roots of the characteristic equation $|\Delta-| I_{4} \mid=0$. For cholesterics in general, and for SmC* at normal incidence, we have $\Delta_{11}=\Delta_{13}=0$ and the roots are given by :

$$
l_{1,2,3,4}= \pm\left[\frac{1}{2}\left[\Delta_{43}+\Delta_{12} \Delta_{21} \pm\left(\left(\Delta_{43}-\Delta_{12} \Delta_{21}\right)^{2}+4 \Delta_{12} \Delta_{23}^{2}\right)^{1 / 2}\right]\right]^{1 / 2} .
$$

For the $\mathrm{SmC}^{*}$ case at oblique incidence, we found the roots numerically.

\section{The boundary problem.}

In order to express the reflection coefficients in terms of the characteristic matrix elements, we assume that the sample is sandwiched between two glass plates with refractive index $\sqrt{\varepsilon_{0}}$. A beam of linearly polarized light strikes the lower one (Fig. 1) at an angle $\varphi$ relative to the $z$ direction with amplitudes $A_{\|}, A_{\perp}$, parallel and perpendicular to the plane of incidence, respectively. If corresponding reflected and transmitted amplitudes are $R_{\|}, R_{\perp}$ and $T_{\|}, T_{\perp}$, then the field components are given by :

$$
\begin{aligned}
& E_{x}^{(\mathbf{i})}=-A_{\|} \cos \varphi \cdot \mathrm{e}^{-i \tau_{\mathrm{i}}} ; \quad E_{y}^{(\mathbf{i})}=A_{\perp} \mathrm{e}^{-i \tau_{\mathrm{i}}} ; \quad H_{x}^{(\mathbf{i})}=A_{\perp} \cos \varphi \cdot \mathrm{e}^{-i \tau_{1}} ; \\
& H_{y}^{(\mathrm{i})}=-A_{\|} \cdot \sqrt{\varepsilon_{0}} \cdot \mathrm{e}^{-i \tau_{\mathrm{i}}} ; \quad \tau_{\mathrm{i}}=\omega t-k_{\mathrm{i}} \cdot \underline{r} \\
& E_{x}^{(t)}=-T_{\|} \cos \varphi \cdot \mathrm{e}^{-i \tau_{\mathrm{t}}} ; \quad E_{y}^{(\mathfrak{t})}=T_{\perp} \cdot \mathrm{e}^{-i \tau_{\mathrm{t}}} ; \quad H_{x}^{(\mathrm{t})}=-T_{\perp} \cos \varphi \cdot \sqrt{\varepsilon_{0}} \cdot \mathrm{e}^{-i \tau_{\mathrm{t}}} ; \\
& H_{y}^{(t)}=T_{\|} \cdot \sqrt{\varepsilon_{0}} \cdot \mathrm{e}^{-i \tau_{\mathrm{t}}} ; \quad \tau_{\mathrm{t}}=\omega t-k_{\mathrm{t}} \cdot \underline{r} \\
& E_{x}^{(\mathrm{r})}=-R_{\|} \cos \varphi \cdot \mathrm{e}^{-i \tau_{\mathrm{r}}} ; \quad E_{y}^{(\mathrm{r})}=R_{\perp} \cdot \mathrm{e}^{-i \tau_{\mathrm{r}}} ; \quad H_{x}^{(\mathrm{r})}=-R_{\perp} \cos \varphi \cdot \sqrt{\varepsilon_{0}} \cdot \mathrm{e}^{-i \tau_{\mathrm{r}}} ; \\
& H_{y}^{(\mathrm{r})}=-R_{\|} \cdot \sqrt{\varepsilon_{0}} \cdot \mathrm{e}^{-i \tau_{\mathrm{r}}} ; \quad \tau_{\mathrm{r}}=\omega t-k_{\mathrm{r}} \cdot \underline{r} .
\end{aligned}
$$

The continuity requirement of the tangential components on the boundary gives

$$
\begin{array}{ll}
E_{x}^{(\mathbf{i})}+E_{x}^{(\mathrm{r})}=E_{x}^{(\mathbf{l})} ; & E_{y}^{(\mathbf{i})}+E_{y}^{(\mathrm{r})}=E_{y}^{(\mathbf{t})} \\
H_{x}^{(\mathbf{i})}+H_{x}^{(\mathrm{r})}=H_{x}^{(\mathbf{l})} ; & H_{y}^{(\mathbf{(})}+H_{y}^{(\mathrm{r})}=H_{y}^{(\mathbf{t})}
\end{array}
$$


then

$$
\left(\psi_{\mathrm{r}}+\psi_{\mathrm{i}}\right)=M(L) \cdot \psi_{\mathrm{t}}
$$

$L=I N h$ is the sample thickness. Our calculated spectra are taken from the solution of this set of equations (12) and we get :

$$
\begin{array}{ll}
R_{\mathrm{PP}}=\left|R_{\|} / A_{\|}\right|^{2} ; & R_{\mathrm{PS}}=\left|R_{\perp} / A_{\|}\right|^{2} \\
R_{\mathrm{SS}}=\left|R_{\perp} / A_{\perp}\right|^{2} ; & R_{\mathrm{SP}}=\left|R_{\|} / A_{\perp}\right|^{2} .
\end{array}
$$

\section{Results and discussion.}

Following Berreman [20], we took the dielectric constants to be $\varepsilon_{1}=\varepsilon_{2}=2.0, \varepsilon_{3}=3.0$, and for the glass plates $\varepsilon_{0}=2.3$.

Then for comparison purposes we took the same dielectric constants, but with $\varepsilon_{0}=1.0$. The number of periods in the sample is $N=10$ and every period is taken to be equal to $I=500$ layers which seems to be reasonable, since the peak heights increase monotonically and the difference in heights between $I=500$ and $I=1000$ was only 0.01 , while between $I=500$ and $I=100$ it was close to 0.04 , which cannot be neglected.

4. 1 Normal INCIDENCE CASE. - Figure 2 shows the reflectance spectra at normal incidence. The symbols $\mathrm{P}$ and S replace $\pi$ and $\sigma$ in reference [20], for example $R_{\mathrm{PS}}$ designates the reflection as measured by an

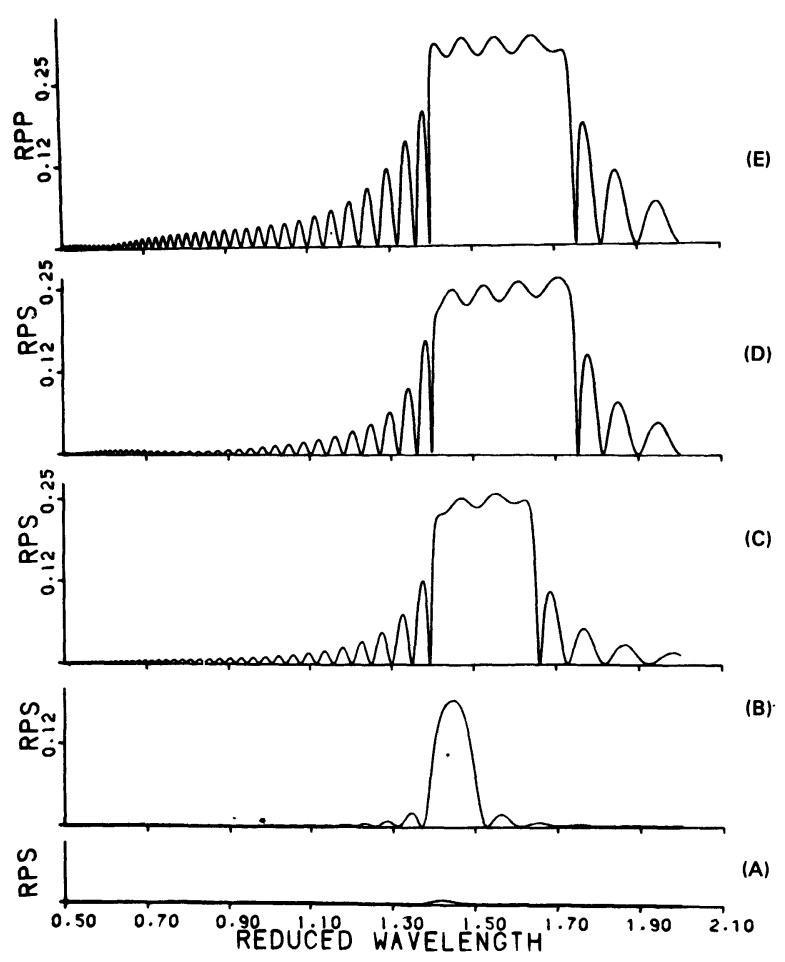

Fig. 2. - Reflectance spectra vs. reduced wavelength for $\mathbf{P}$ orientation, observed through a plane polarizing analyser in $\mathrm{S}$ orientation, that is $\boldsymbol{R}_{\mathrm{PS}}$ at normal incidence, for tilt angles (A) $10^{\circ}$, (B) $30^{\circ}$, (C) $60^{\circ}$, (D) $90^{\circ}$ and (E) $R_{\mathrm{PP}}$ at $\theta .=90^{\circ}$, with $\varepsilon_{0}=2.3$. analyser rotated perpendicular to the plane of incidence when the incident light is polarized parallel to the plane of incidence. At normal incidence we obtain one peak which could be considered as first order Bragg reflection peak for cholesterics and second order for $\mathrm{SmC}^{*}$. An interesting property seen in this case is the existence of oscillations inside

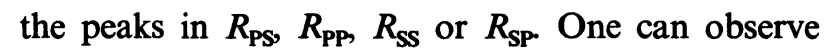
that the maxima in $R_{\mathrm{PP}}$ coincide with the minima in $R_{\mathrm{PS}}$ and so it is between $R_{\mathrm{SS}}$ and $R_{\mathrm{SP}}$. That is to say, there is energy interchange between $R_{\mathrm{PP}}$ and $R_{\mathrm{PS}}$ or $R_{\mathrm{SS}}$ and $\boldsymbol{R}_{\mathrm{SP}}$. In the sum spectra (Fig. 3) the oscillations disappear, but the peak still consists of two branches distinguishable in the calculated data, but the difference in their heights is so small that it is difficult to see them in the figure.

The tilt dependence of the peak height and width at half the maximum is shown in figures 4 and 5 . The height, the width and the number of oscillations increase with increasing $\theta$. The increase of the height and the width with $\theta$ could be referred to the increase in the birefringence, while the increase in the number of oscillations have no simple interpretation for the moment.

4.2 Oblique InCiDENCE CASE. - Figures 6-9 show the reflection spectra at different incidence and tilt angles. All reflection orders appear in this case and an additional sequence of reflection peaks appears in $R_{\mathrm{PS}}$ and $R_{\mathrm{SP}} \mathrm{SmC}^{*}$ spectra, but not in $R_{\mathrm{PP}}$ and $R_{\mathrm{SS}}$ and not in cholesterics.

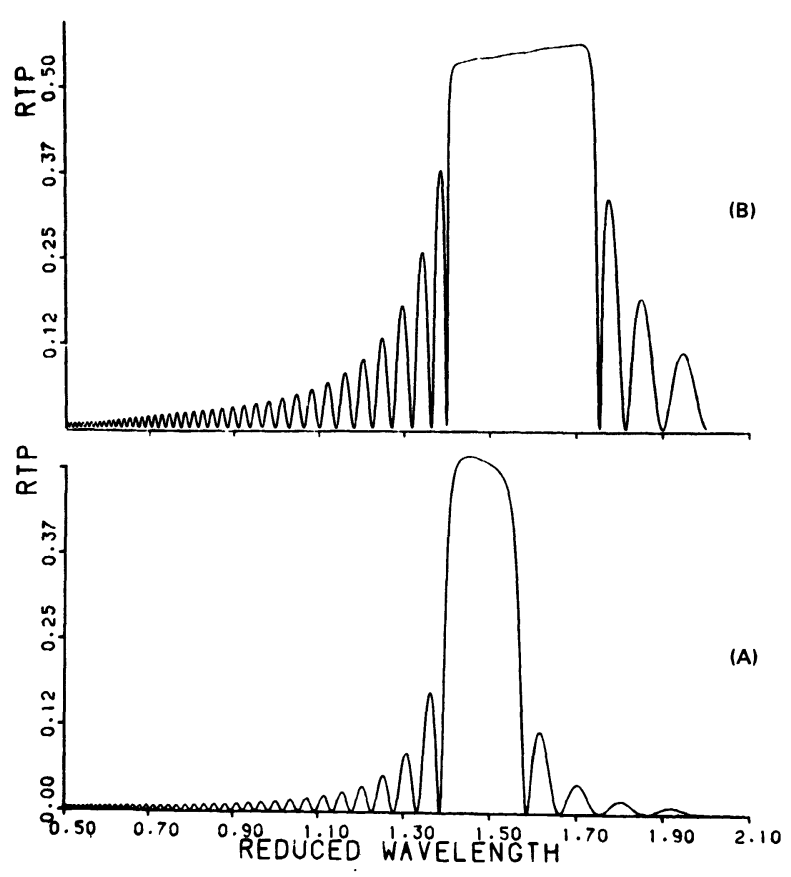

Fig. 3. - Total reflection spectra vs. reduced wavelength for $P$ orientation at normal incidence with tilt angles (A) $45^{\circ}$, (B) $90^{\circ}$, with $\varepsilon_{0}=2.3$. 


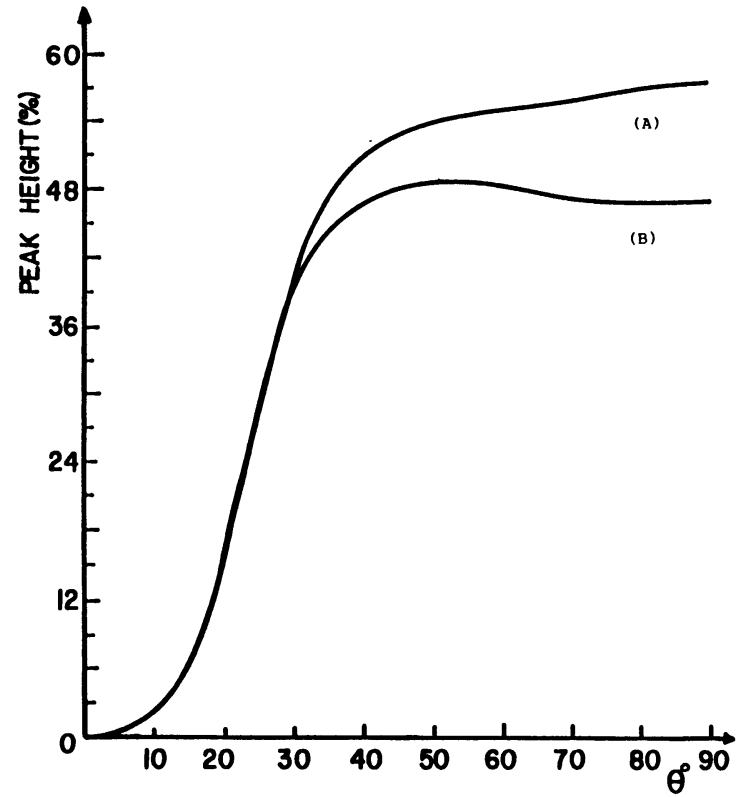

Fig. 4. - Tilt dependence of the total reflection peak height at normal incidence with (A) S orientation (B) $P$ orientation, with $\varepsilon_{0}=2.3$.

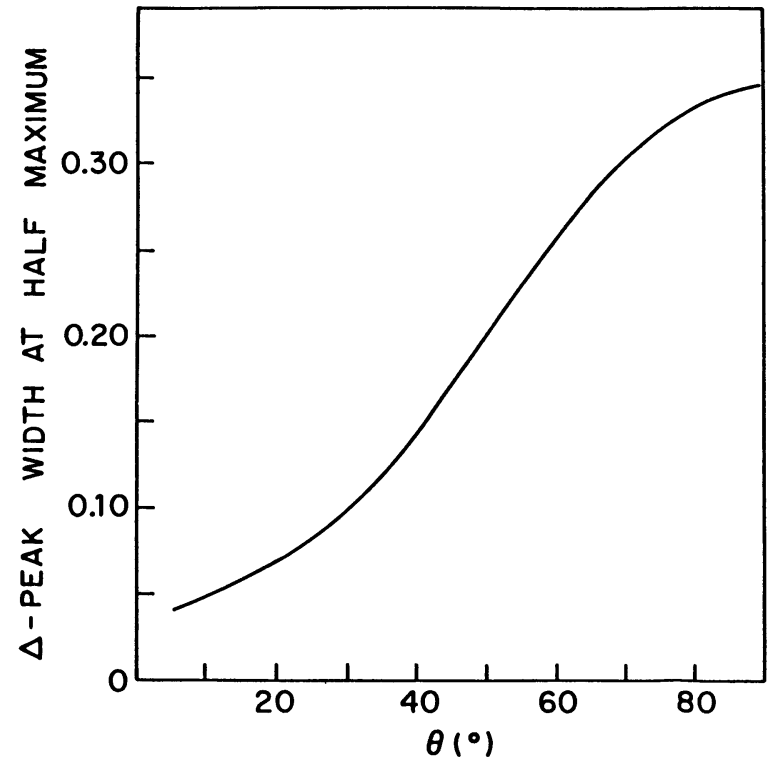

Fig. 5. - Tilt dependence of the total reflection peak width at half maximum both for $\mathbf{P}$ and $\mathrm{S}$ orientation at normal incidence, with $\varepsilon_{0}=2.3$.
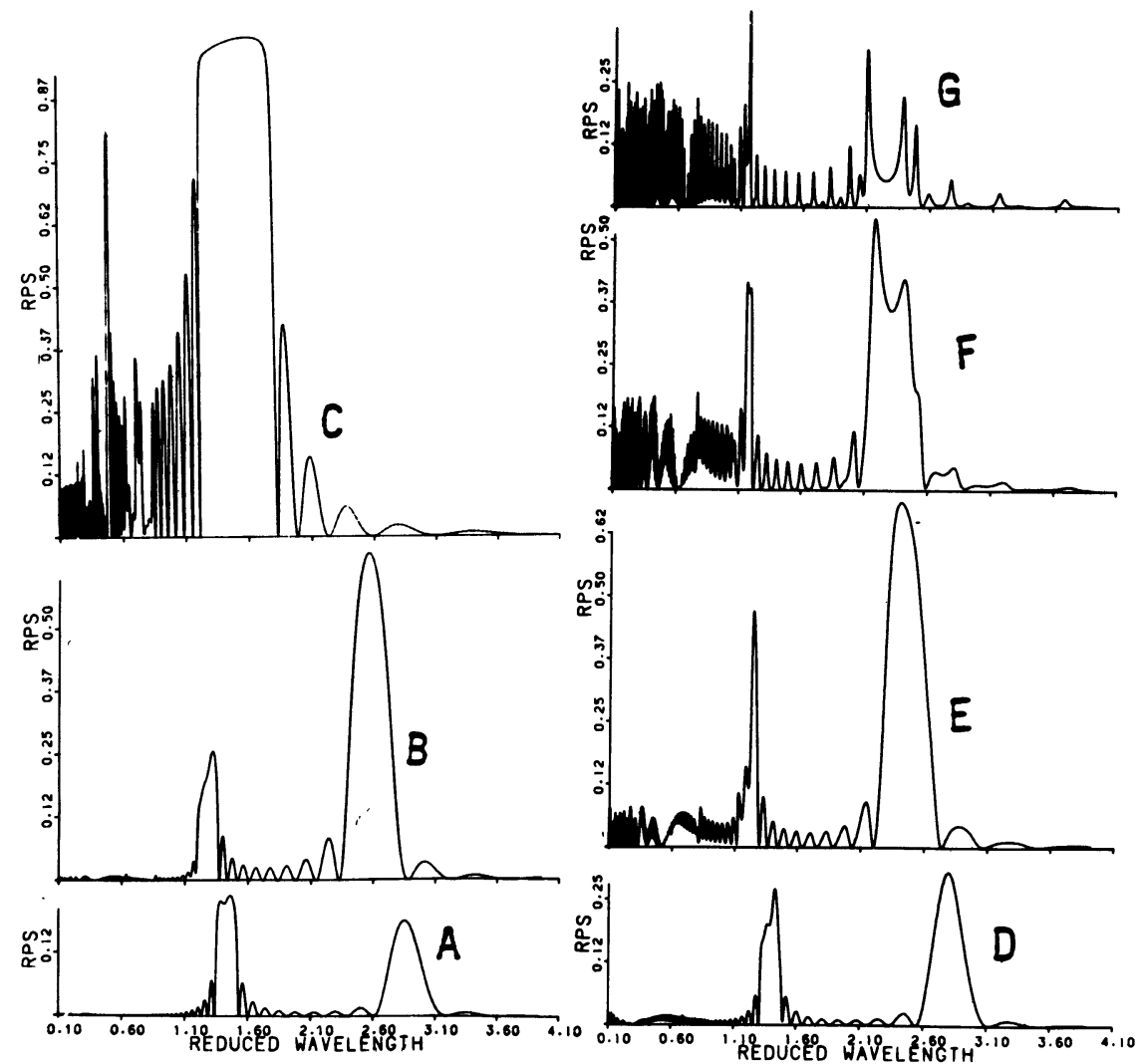

Fig. 6. $-R_{\mathrm{PS}}$ spectra at $\theta=45^{\circ}$ and different angles of incidence $\varphi$ for $\varepsilon_{0}=2.3:$ (A) $15^{\circ}$, (B) $30^{\circ}$, (C) $60^{\circ}$ and for $\varepsilon_{0}=1.0$ (D) $30^{\circ}$, (E) $60^{\circ}$, (F) $75^{\circ}$, (G) $85^{\circ}$. 


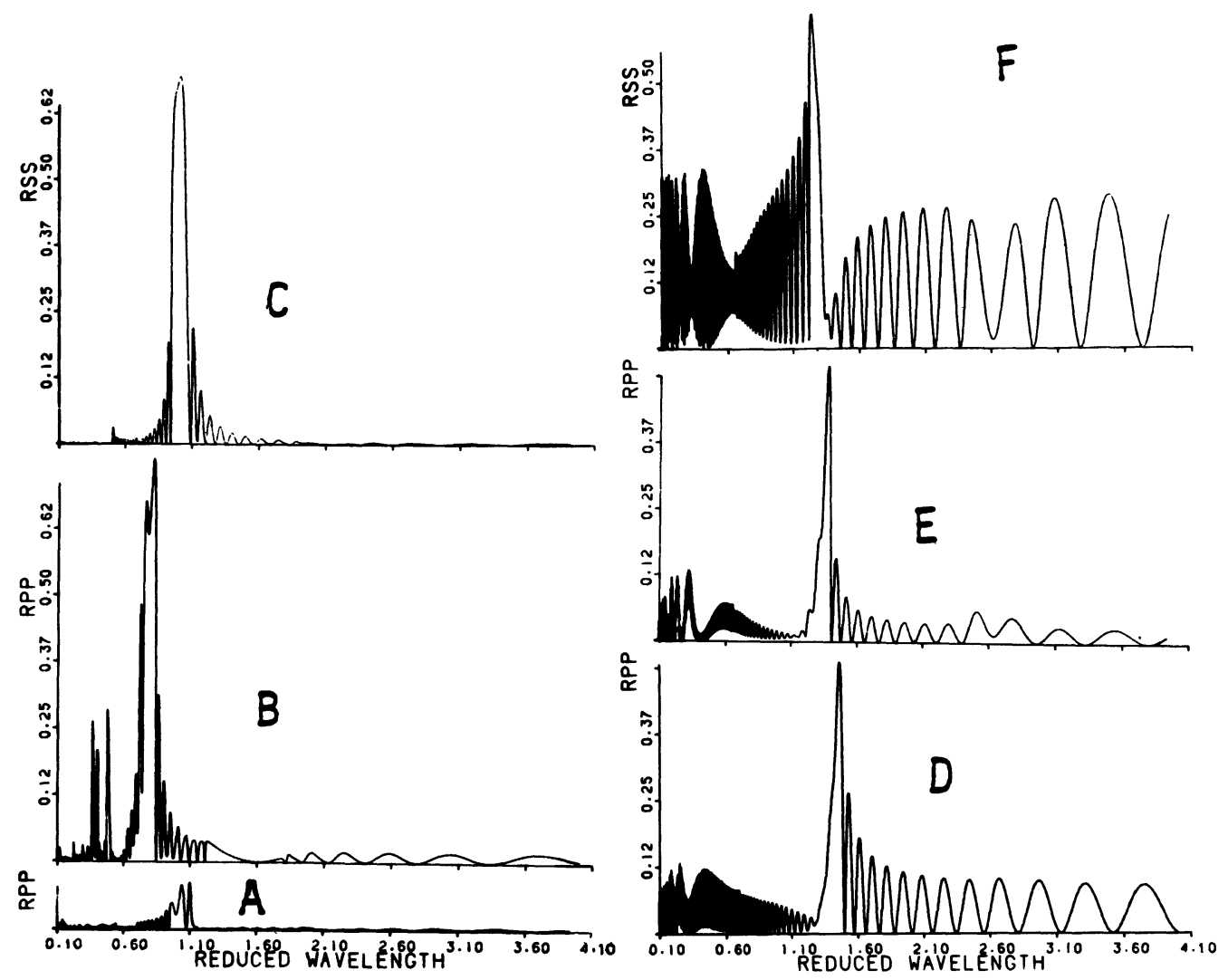

Fig. 7. $-R_{\mathrm{PP}}$ spectra at tilt angle $\theta=45^{\circ}$ and various angles of incidence $\varphi$ with $\varepsilon_{0}=2.3:$ (A) $45^{\circ}$, (B) $60^{\circ}$ and (C) $R_{\mathrm{Ss}}$ at $\theta=\varphi=45^{\circ}$ and $R_{\mathrm{PP}}$ for $\varepsilon_{0}=1.0:$ (D) $\varphi=30^{\circ}$, (E) $\varphi=45^{\circ}$ and (F) $R_{\mathrm{SS}}$ at $\theta=\varphi=45^{\circ}$.

In figure 11 the full pitch peak height and width dependence on tilt angle as taken from figures 6-9 are given, showing that there exists some angle where the peak height and width reach a maximum value. Then they start decreasing.

A similar behaviour of the full pitch dependence on the incident angle seems to exist, but since it starts splitting after some incidence angle, then the peak height and width are not well defined, so we omit such curves. We are not sure whether these angles are related to the complete reflection region for the first order in cholesterics as predicted by Dimitrienko and Belyakov [6, 7]. Let us call this angle the turning point tilt or turning point incident angle, respectively. Those angles do not depend on the outer dielectric constant $\varepsilon_{0}$ as seen in figure 11. Furthermore we checked that the turning point tilt angle does not depend on the incidence angle, and conversely the turning point incidence angle does not depend on the tilt angle.

The energy interchange phenomenon observed in the normal incidence case is also clear in the half pitch peak (second order), and the number of oscillations increases with the tilt and incident angles. The sum spectra show that this peak is composed of two branches at small incident angles and it reaches three at larger incidence angles.

The phenomenon is stronger in the case of $\varepsilon_{0}=1.0$ than that with $\varepsilon_{0}=2.3$. This could indicate that it originates from the effect of the reflections from the. boundarjes on the reflection peak, which are stronger for the case with $\varepsilon_{0}=1.0$ than with $\varepsilon_{0}=2.3$.

Every branch becomes narrower and the average peak height increases with increasing incident angle $\varphi$. The width at half maximum increases with the tilt and has the same behaviour as in the normal incidence case. So does the maximum peak height, but it reaches more than $50 \%$ - the fact which indicates that it is not circularly polarized. The centre of the peak shifts toward the long wavelength region with increasing tilt, but toward short wavelength with increasing $\varphi$.

The centre position of the full reflection peak shifts toward the short wavelength region with increasing angle of incidence, while it shifts toward long wavelength with increasing tilt angle. The former is easily interpreted according to Bragg's law of diffraction; however the latter has no simple interpretation for the moment. 


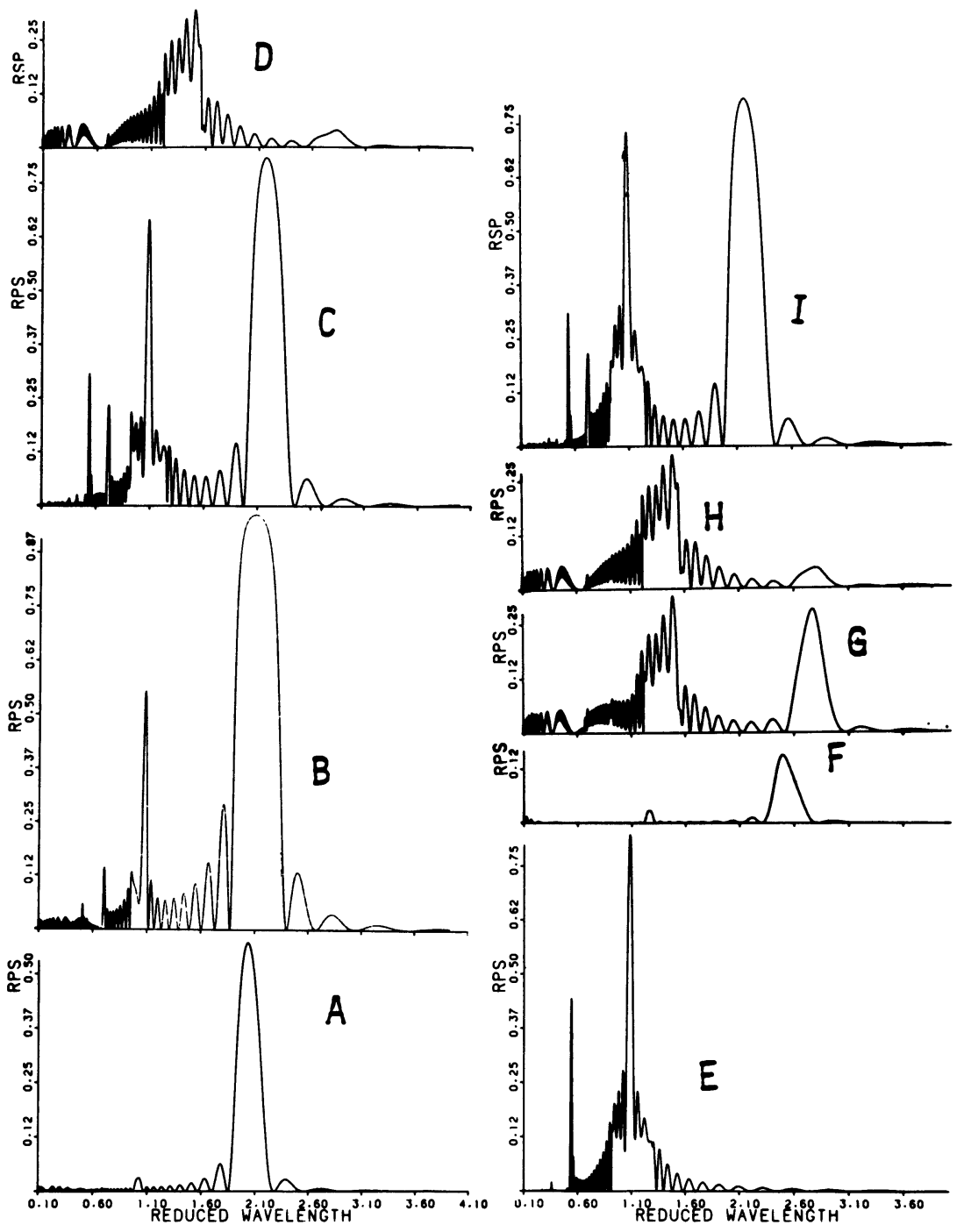

Fig. 8. $-R_{\mathrm{PS}}$ spectra at incidence angle $\varphi=45^{\circ}$ and various tilt angles with $\varepsilon_{0}=2.3:$ (A) $15^{\circ}$, (B) $45^{\circ}$, (C) $75^{\circ}$, (E) $90^{\circ}$ and (I) $R_{\mathrm{SP}}$ at $\theta=75^{\circ}$ and $R_{\mathrm{PS}}$ for $\varepsilon_{0}=1.0:$ (F) $15^{\circ},(\mathrm{G}) 75^{\circ},(\mathrm{H}) 85^{\circ}$ and (D) $R_{\mathrm{SP}}$ at $\theta=85^{\circ}$.
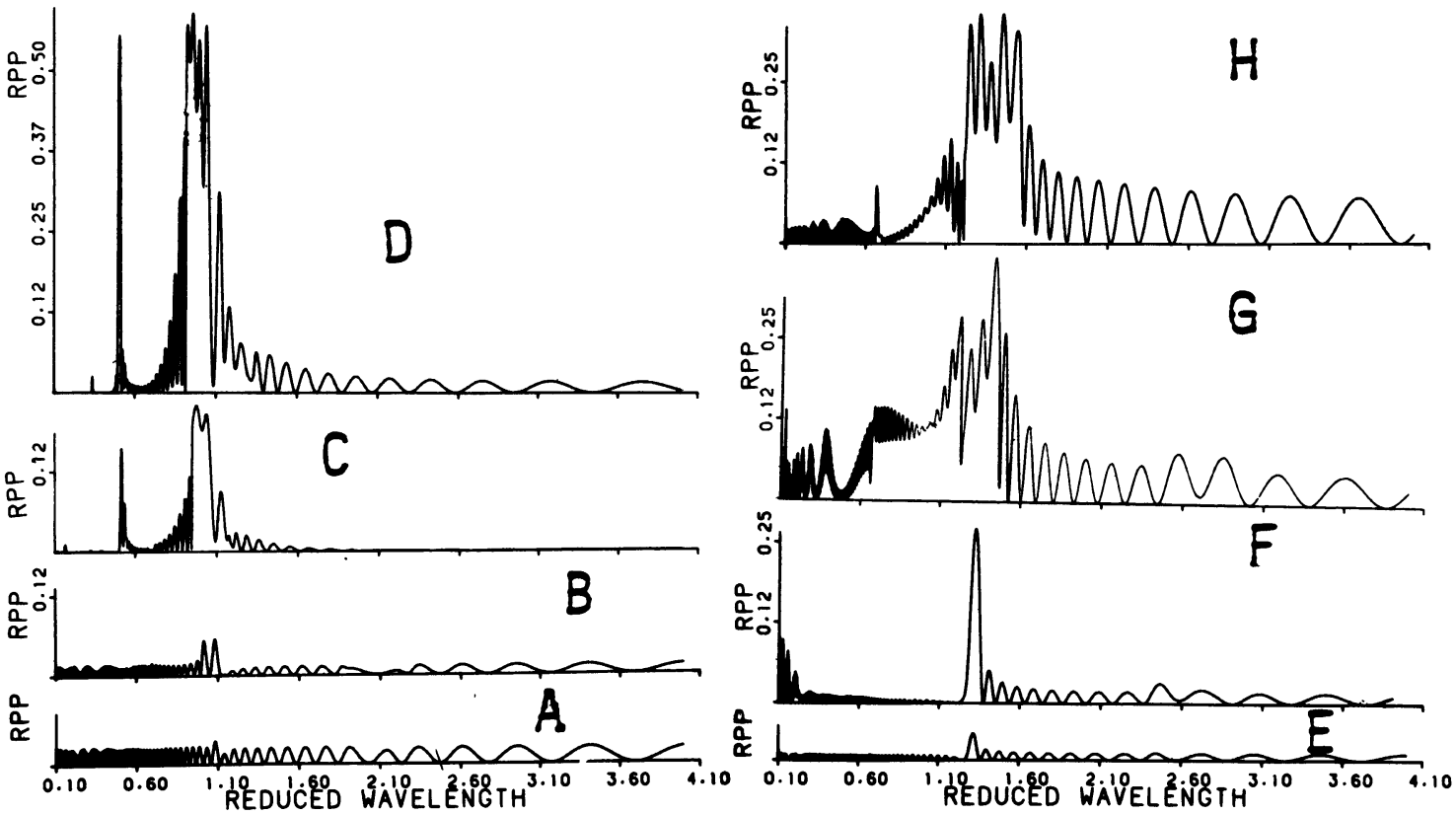

Fig. 9. $-R_{\mathrm{PP}}$ spectra at incidence angle $\varphi=45^{\circ}$ and different tilt angles for $\varepsilon_{0}=2.3:$ (A) $15^{\circ}$, (B) $30^{\circ}$, (C) $60^{\circ}$, (D) $90^{\circ}$ and for $\varepsilon_{0}=1.0(\mathrm{E}) 15^{\circ},(\mathrm{F}) 30^{\circ},(\mathrm{G}) 60^{\circ},(\mathrm{H}) 90^{\circ}$. 


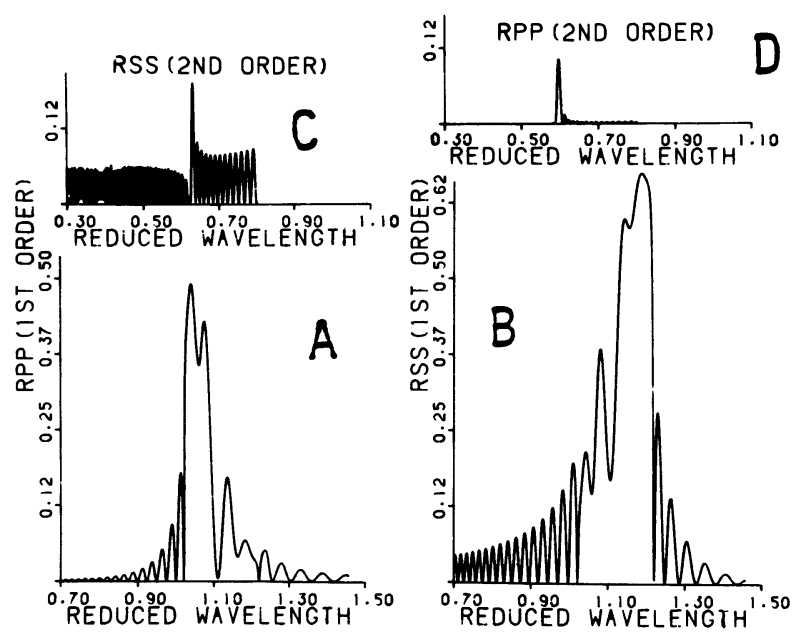

Fig. 10. - Reflection spectra calculated for comparison with reference $[17,19]$. Different principal dielectric constants were taken for the 1st and 2 nd order peaks : (A) $R_{\mathrm{PP}}$ for the 1 st order, (B) $R_{\mathrm{SS}}$ for the 1 st order, (C) $R_{\mathrm{SS}}$ for the 2 nd order, (D) $R_{\mathrm{PP}}$ for the 2 nd order.

The series expansion approximation up to the second order of Berreman and Scheffer [20] seems to give satisfactory results, as is seen in figures 6-9 for the case $\theta=\varphi=45^{\circ}$ with $\varepsilon_{0}=2.3$. But the expansion up to the 1st order is not sufficient [18]. This fact is probably responsible for the discrepancy between the measured and calculated reflection spectra done by Berreman and Scheffer [17] on the cholesteric phase of $2 \mathrm{MBAB}$. This is seen in figure 10, especially for the $R_{\mathrm{SS}}$ spectra. Its height is 0.67 , while the one predicted by Berreman and Scheffer [17] is 0.80 and the experimental one is 0.62. Later, Berreman [18] became aware of the fact that the expansion up to the 1 st power is not sufficient and it causes a cumulative error when one performs so large a number of $4 \times 4$ matrix multiplications and one raises the result to the $N$ th power.

The existence of the energy interchange phenomena between $R_{\mathrm{PS}}$ and $R_{\mathrm{PP}}$ or $R_{\mathrm{SP}}$ and $R_{\mathrm{SS}}$ spectra proves that the beams parallel and perpendicular to the plane of incidence are not two independent waves as is the case in isotropic media.

The subsidiary oscillations seen in $R_{\mathrm{PP}}$ and $R_{\mathrm{SS}}$ are due to multiple reflections from the boundaries. This we prove by calculating. the spectra for $\theta=0^{\circ}$ in figure 9. We see that these reflections still exist while in $R_{\mathrm{PS}}$ and $R_{\mathrm{SP}}$ we get nothing which is a known property of isotropic media.

Other interesting phenomena seen in the short wavelength range are the beat structure of $R_{\mathrm{PP}}$ and $R_{\mathrm{SS}}$ spectra and the swells in $R_{\mathrm{PS}}$ and $R_{\mathrm{SP}}$. The phenomena are clearer in the spectra with $\varepsilon_{0}=1.0$ than that with $\varepsilon_{0}=2.3$. The reason is probably the difference in the subsidiary oscillations, which are stronger in the former case than in the latter. An enlarged

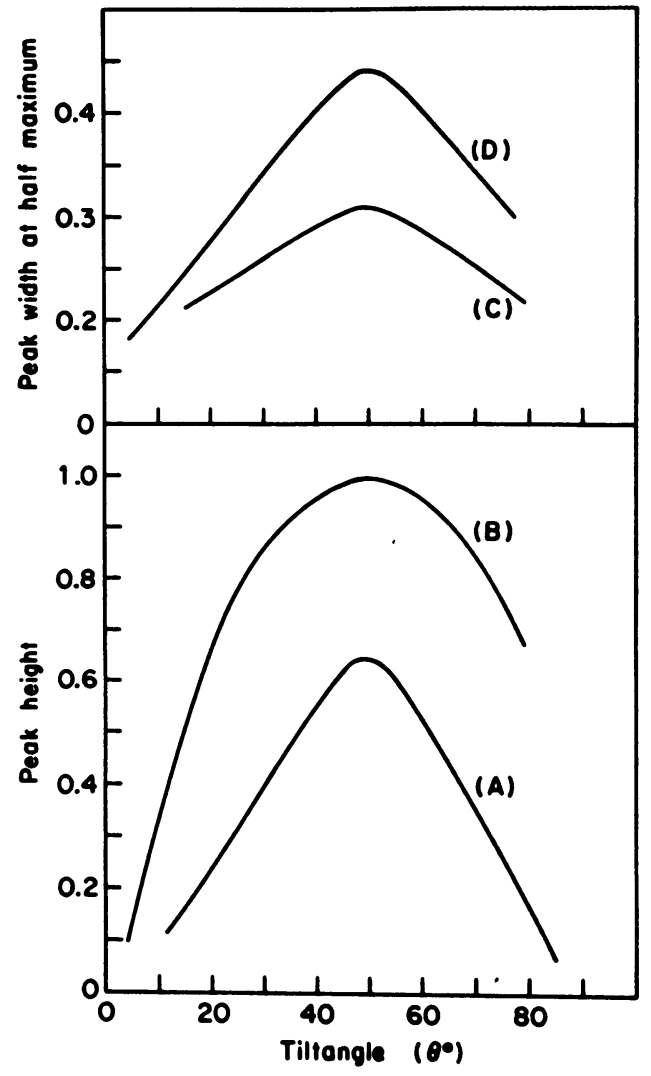

Fig. 11. $-R_{\mathrm{PS}}$ full pitch peak dependence on the tilt angle at $\varphi=45^{\circ}$ with $\varepsilon_{0}=1.0$ (A) peak height, (C) peak width and with $\varepsilon_{0}=2.3:$ (B) peak height, (D) peak width.

scale for clarifying the structure of the swells and beats is given in figure 13 .

The existence of beat structure could be attributed to the superposition of two waves with different wavelengths or phases and swells are due to two waves with different amplitudes and different phases.

The phenomenon is not seen in the normal incidence case (too small), and, by increasing the incident angle, beats increase rapidly while swells slowly with the appearence of two additional waves superimposed on them. This seems to start at the turning point incident angle and it reaches a fine structure at higher angles of incidence. By increasing the tilt angle, beats increase, reaching a fine structure and at the turning point angle they approach each other closely resulting in some deformation. Swells, on the other hand, increase with the tilt until the turning tilt angle where they start decreasing.

A mysterious property seen in the spectra presented is that the peaks are higher for the case with $\varepsilon_{0}=2.3$ than those with $\varepsilon_{0}=1.0$.

Higher order peaks are seen in the spectra, a peak corresponding to one third of the full pitch appears at $R_{\mathrm{SP}}$ and $R_{\mathrm{PS}}$ for $\mathrm{SmC}^{*}$, but not in cholesterics and not in $R_{\mathrm{SS}}$ and $R_{\mathrm{pp}}$. It has a narrow width and it changes slowly with $\varphi$ and $\theta$. It appears that a com- 

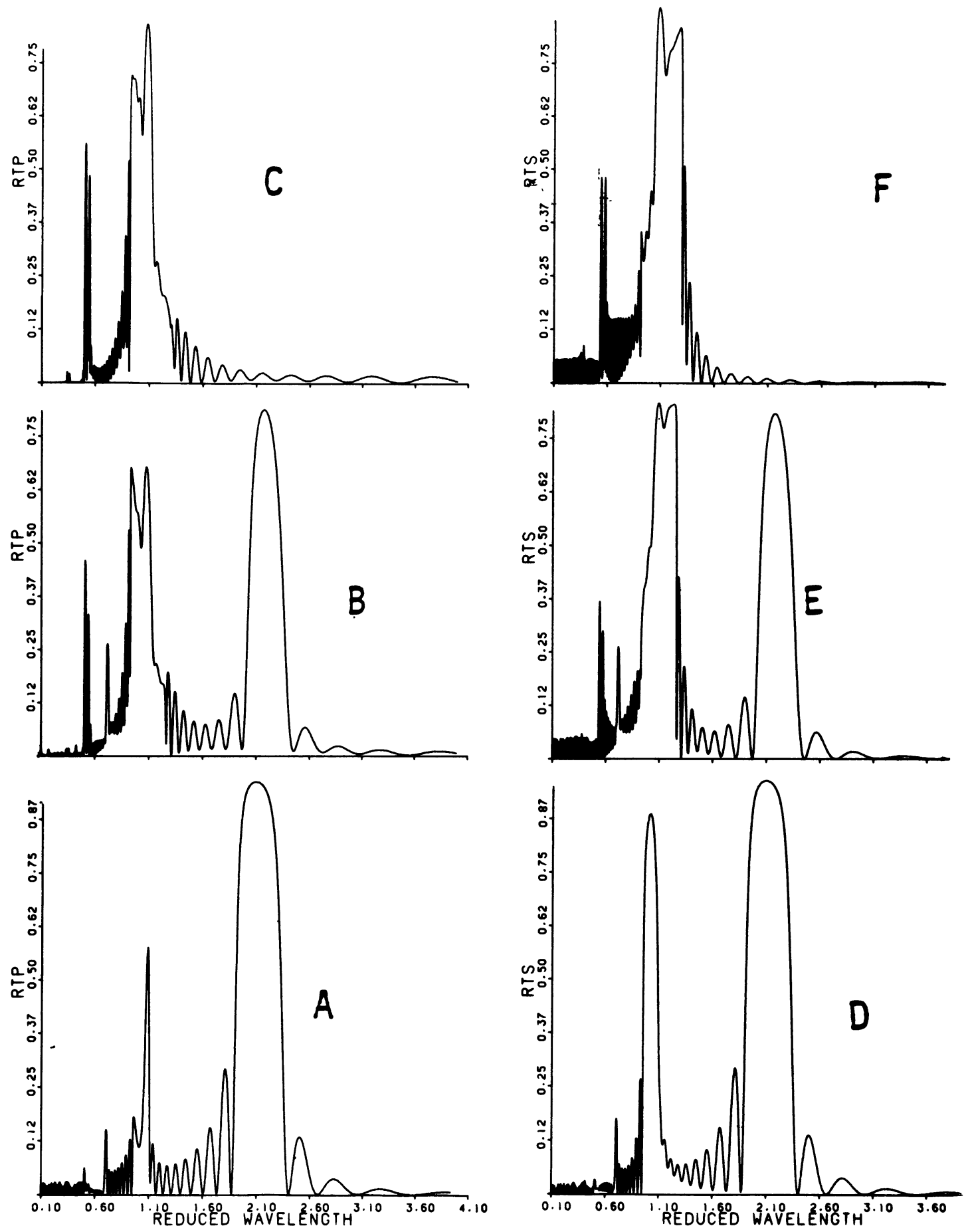

Fig. 12. - Total reflection spectra at $\varphi=45^{\circ}$ and various tilt angles for $P$ orientation $R_{\mathrm{TP}}$ (A) $45^{\circ}$, (B) $75^{\circ}$, (C) $90^{\circ}$ and for $S$ orientation $R_{\mathrm{TS}}$ (D) $45^{\circ}$, (E) $75^{\circ}$, (F) $90^{\circ}$, all with $\varepsilon_{0}=2.3$.

plete set of reflections exists which corresponds to odd parts of the full pitch and has the same polarization properties as the full pitch band, but their width and height decrease rapidly with increasing order. A fourth order peak appears both for $\mathrm{SmC}^{*}$ and cholesterics, it is stronger in $R_{\mathrm{PP}}$ and $R_{\mathrm{SS}}$ than in
$R_{\mathrm{PS}}$ and $R_{\mathrm{SP}}$, its height increases with $\theta$ and $\varphi$, but the centre seems to be constant.

In conclusion : the exact calculation of reflectance spectra for helicoidal liquid crystals using the $4 \times 4$ characteristic matrix method is necessary to obtain the real picture, especially for the oblique incidence 

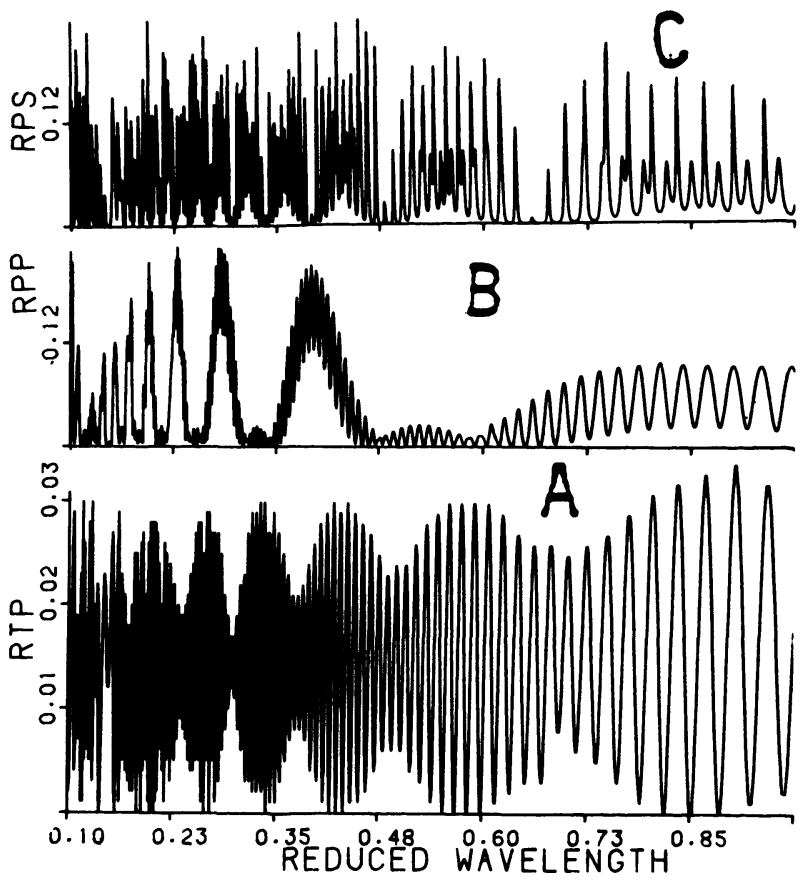

Fig. 13. - Enlarged scale for reflection in the short wavelength region (A) $R_{\mathrm{TP}}$ at $\varphi=45^{\circ}, \theta=15^{\circ}, \varepsilon_{0}=2.3$; (B) $R_{\mathrm{PP}}$ at $\varphi=60^{\circ}, \theta=45^{\circ}, \varepsilon_{0}=1.0$; (C) $R_{\mathrm{PS}}$ at $\varphi=85^{\circ}$, $\theta=45^{\circ}, \varepsilon_{0}=1.0$. case. The exact calculation yields additional features, not seen in an approximate calculation, and a number of discrepancies with the results of other workers [6$13,17-20]$, concerning peak heights, number of branches and dependence on tilt and incident angles.

More calculations, including these for incident circularly polarized light, the higher order reflections, the polarization properties of the reflected peaks and an interpretation of the results, will be given by us in a separate paper. Furthermore we wish to point out that we have verified experimentally the results of figures 4 and 5 on DOBAMBC and these results will be published shortly.

\section{Acknowledgments.}

We would like to thank Prof. C. Oldano for his note that $\sqrt{\varepsilon_{0}}$ has to appear near to $\sin \varphi$ in the elements of the $\Delta$-matrix and one of the referees who called our attention to references [11-14].

\section{References}

[1] De Vries, H. L., Acta Cryst. 4 (1951) 219.

[2] Marathay, A. S., J. Opt. Soc. Am. 61 (1971) 1363.

[3] Chandrasekhar, S. and Srinivasarao, K. N., Acta Crystallogr. A 24 (1968) 445.

[4] Nityananda, R., Mol. Cryst. Liq. Cryst. 21 (1973) 315-331.

[5] Parodi, O., J. Physique Colloq. 36 (1975) C1-22.

[6] Belyakov, V. A. and Dimitrienko, V. D., Sov. Phys. Solid State 15 (1974) 1811.

[7] Dimitrienko, V. E. and Belyakov, V. A., Sov. Phys. Solid State 15 (1974) 2365.

[8] Taupin, D., J. Physique Colloq. 30 (1969) C4-32.

[9] Dreher, R., Meier, G. and Saupe, A., Mol. Cryst. Liq. Cryst. 13 (1971) 17.

[10] Dreher, R., Meier, G., Phys. Rev. A 8 (1973) 1616.

[11] Saupe, A., Meier, G., Phys. Rev. A 27 (1983) 2196.

[12] Oldano, C., Miraldi, E., Taverna Valabrega, P., Phys. Rev. A 27 no 6 (1983) 3291.

[13] Miraldi, E., Oldano, C., Taverna, P. I., Trossi, L., Mol. Cryst. Liq. Cryst. C 103 (1983) 155.

[14] Patterson, M. A., Phys. Rev. A 27 no 1 (1983) 520.
[15] Abeles, F., Ann. Phys. Fr. 5 (1950) 777.

[16] Teitler, S. and Heneves, B. W., J. Opt. Soc. Am. 60 6 (1970) 830.

[17] Berreman, D. W., Scheffer, T. J., Phys. Rev. A 25 (1970) 577.

[18] Berreman, D. W., J. Opt. Soc. Am. 62 (1972) 502.

[19] Berreman, D. W., Scheffer, T. J., Mol. Cryst. Liq. Cryst. 11 (1970) 395.

[20] Berreman, D. W., Mol. Cryst. Liq. Cryst. 22 (1973) 175.

[21] Helfrich, W., Сhan, OH, S., Mol. Cryst. Liq. Cryst. 14 (1971) 289.

[22] Takezoe, H., Yokioouchi, Hara, M., Fokuda, A. and EIICHIKUZe, Jpn J. Appl. Phys. 227 (1983) 1080.

[23] Born, M., Wolf, E., Principles of Optics (London, Pergamon Press) 1959, p. 66, 39.

[24] Gantmacher, F. R., Theory of Matrices (Chelsea Publishing Company, U.S.A.) 1960, Vol. 1, Ch. 5.

[25] Goldstein, H., Classical Mechanics (Second Edition) 1981, p. 133. 